TITLE:

\title{
Impact of preoperative uncontrollable hepatic hydrothorax and massive ascites in adult liver transplantation.
}

\section{AUTHOR(S):}

Endo, Kosuke; lida, Taku; Yagi, Shintaro; Yoshizawa, Atsushi; Fujimoto, Yasuhiro; Ogawa, Kohei; Ogura, Yasuhiro; Mori, Akira; Kaido, Toshimi; Uemoto, Shinji

\section{CITATION:}

Endo, Kosuke ... [et al]. Impact of preoperative uncontrollable hepatic hydrothorax and massive ascites in adult liver transplantation.. Surgery today 2014, 44(12): 2293-2299

\section{ISSUE DATE:}

2014-12

URL:

http://hdl.handle.net/2433/202913

\section{RIGHT:}

The final publication is available at Springer via http://dx.doi.org/10.1007/s00595-014-0839-y; The full-text file will be made open to the public on 08 February 2015 in accordance with publisher's 'Terms and Conditions for Self-Archiving', This is not the published version. Please cite only the published version.; この論文は出版社版でありません。引用の際 には出版社版をご確認ご利用ください。 
1 Impact of Preoperative Uncontrollable Hepatic Hydrothorax and Massive Ascites

2 in Adult Liver Transplantation

3

4 Kosuke Endo, Taku Iida, Shintaro Yagi, Atsushi Yoshizawa, Yasuhiro Fujimoto, Kohei

5 Ogawa, Yasuhiro Ogura, Akira Mori, Toshimi Kaido, and Shinji Uemoto

6

7 Division of Hepato-Biliary-Pancreatic and Transplant Surgery, Department of Surgery,

8 Graduate School of Medicine, Kyoto University

9

10 Corresponding author: $\mathrm{T}$. Iida, $\mathrm{MD}, \mathrm{PhD}$.

11 Division of Hepato-Biliary-Pancreatic and Transplant Surgery, Department of Surgery,

12 Graduate School of Medicine, Kyoto University, 54 Kawahara-cho, Shogoin, Sakyo-ku,

13 Kyoto 606-8507, Japan

14 E-mail address: itaku@kuhp.kyoto-u.ac.jp

15 Telephone: +81-75-751-4323

16 Fax number: $+81-75-751-4348$

17

18 Article type: Original article (Clinical original)

19

20 Key words: hepatic hydrothorax, liver transplantation, massive ascites, bacteremia, 21 mortality. 
1 Abstract:

2 Purpose: Uncontrollable hepatic hydrothorax and massive ascites (H\&MA) requiring

3 preoperative drainage are sometimes encountered in liver transplantation (LT). We

4 retrospectively analyzed the characteristics of such patients and the impact of H\&MA

$5 \quad$ on the postoperative course.

6 Methods: We evaluated 237 adult patients who underwent LT in our institute between

$7 \quad$ April 2006 and October 2010.

8 Results: Recipients with uncontrollable H\&MA (group HA: $n=36$ ) had more

9 intraoperative bleeding, higher Child-Pugh scores, lower serum albumin concentrations,

10 and higher blood urea nitrogen concentrations than those without uncontrollable H\&MA

11 (group C: $n=201$ ). They were also more likely to have preoperative hepatorenal

12 syndrome and infections. The incidence of postoperative bacteremia was higher (55.6\%

13 versus $46.7 \%, P=0.008$ ) and the 1 - and 3-year survival rates were lower ( 1 year: $58.9 \%$

14 versus $82.9 \%$; 3 years: $58.9 \%$ versus $77.7 \%$; $P=0.003$ ) in group HA than in group C.

15 Multivariate proportional regression analyses revealed that uncontrollable H\&MA and

16 Child-Pugh score were independent risk factors for the postoperative prognosis.

17 Conclusions: Postoperative infection control may be one of the important means of

18 improving outcome for patients with uncontrollable H\&MA undergoing LT, and

19 clinicians should strive to undertake surgery before H\&MA becomes uncontrollable. 


\section{INTRODUCTION}

2

3 Liver transplantation (LT) is now performed in many countries as a treatment for

4 end-stage liver disease. In the current era, as a result of expansion of the indication of

5 LT for HCC candidates, it also gives us a choice of curative treatment for unresectable

6 hepatocellular carcinoma, and satisfactory long-term outcomes have been achieved [1].

7 However, it still has a relatively high mortality rate compared with other

8 hepatobiliary-pancreatic procedures owing to the potentially poor preoperative

9 condition of the patients, use of immunosuppressive agents, rejection and infection. The

10 preoperative condition of transplant recipients is a particularly important factor that

11 influences the outcome of LT.

We sometimes encounter patients requiring LT with uncontrollable hepatic

13 hydrothorax or massive ascites (H\&MA) that must be drained before surgery; it has also

14 been reported that H\&MA is an independent risk factor for postoperative bacteremia 15 [2].

16 In this study, we focused on preoperative uncontrollable H\&MA in LT

17 candidates, and evaluated the perioperative course of patients who went on to become 18 LT recipients. 
METHODS

2

$3 \quad$ Patients

4 Between April 2006 to October 2010, 237 adult patients underwent LT at Kyoto

5 University Hospital, Japan (227 were living-donor cases, and 10 deceased-donor cases).

6 There were 117 males and 120 females; their median age was 54.9 years (range: 18-69

7 years). The indications for LT in these patients included: hepatocellular carcinoma in 78

8 cases; hepatocellular diseases, such as hepatitis B virus-associated liver cirrhosis,

9 hepatitis C virus-associated liver cirrhosis, and alcoholic liver cirrhosis, in 133 cases;

10 progressive intrahepatic cholestatic diseases, such as primary biliary cirrhosis and

11 primary sclerosing cholangitis, in 29 cases; re-transplantation owing to graft loss in 17

12 cases; fulminant hepatic failure in 17 cases; cryptogenic cirrhosis in 14 cases; biliary 13 atresia after the Kasai operation in eight cases; autoimmune hepatitis in six cases;

14 metabolic liver diseases in six cases; and other causes in seven cases.

16 Antimicrobial prophylaxis

17 Perioperative antimicrobial prophylaxis consisted of cefotaxime (2 g/day intravenously)

18 and ampicillin (4 g/day intravenously) twice daily for 72 hours starting 30 min before

19 surgery. Laxatives were administered as bowel preparation.

21 culturing infection sites or blood. When the focus of the infection was unknown, 
1 broad-spectrum antibiotics were administered empirically. Pre-transplant, antibiotics

2 were given to treat on-going infections such as spontaneous bacterial peritonitis, and

3 pneumonia, for example.

4

\section{Immunosuppression}

6 The standard immunosuppression protocol comprised tacrolimus and a low-dose steroid.

7 We endeavored to maintain the whole blood trough level of tacrolimus between 10 and

$815 \mathrm{ng} / \mathrm{mL}$ during the first 2 weeks, around $10 \mathrm{ng} / \mathrm{mL}$ during the next 2 weeks, and

9 between 5 and $8 \mathrm{ng} / \mathrm{mL}$ thereafter. For the recipients who suffered from side effects of

10 tacrolimus, we changed the immunosuppressant from tacrolimus to cyclosporine micro

11 emulsion.

Steroid therapy with methylprednisolone sodium succinate was initiated at a

13 dose of $10 \mathrm{mg} / \mathrm{kg}$ before graft reperfusion and then tapered from $1 \mathrm{mg} / \mathrm{kg} / \mathrm{day}$ on day 1

14 to $0.3 \mathrm{mg} / \mathrm{kg} /$ day by the end of the first month; this was followed by $0.1 \mathrm{mg} / \mathrm{kg} / \mathrm{day}$ until

15 the end of the third month. Steroid administration was terminated thereafter. In the event

16 of postoperative infection, steroids were discontinued and the target trough level of 
1 tacrolimus was revised downwards.

2

\section{Study Design}

4 The medical records of patients undergoing LT were examined retrospectively to

5 identify those recipients who had required preoperative drainage of uncontrollable

6 H\&MA (allocated to group HA; $n=36$ ) and those recipients who had not (allocated to a

7 control group [group C]; $n=201$ ). Recipients' demographic details, surgical data,

8 occurrence of preoperative hepatorenal syndrome (HRS) and postoperative bacteremia,

9 and patient survival recorded and compared between the groups. Finally, independent

10 prognostic factors for patient survival were evaluated by multivariate analyses.

11

12 Indications for thoracic or intraperitoneal drainage

13 We considered refractory uncontrollable massive ascites to meet the grade 3 with

14 diuretic resistance criterion defined by the International Ascites Club [3]. The diagnosis

15 of hepatic hydrothorax was based on evidence of a large volume effusion (estimated to

16 be $>500 \mathrm{ml}$ ) on chest radiography and/or computed tomography (CT) scans in the

17 absence of underlying pulmonary or cardiac diseases [4]. We used the following indications to guide decisions about when to drain

19 H\&MA before surgery: 1) patients with hydrothorax who remained hypoxic with a

20 peripheral oxygen saturation $\left(\mathrm{SpO}_{2}\right)$ of $\leq 95 \%$ despite supplemental oxygen

21 administration underwent thoracic drainage to improve respiratory function; 2) patients 
1 with hepatorenal syndrome (HRS) underwent peritoneal or thoracic drainage with

2 intravenous albumin supplementation to normalize hemodynamic parameters and

3 prevent the progression of renal dysfunction; and 3) patients experiencing dyspnea,

4 difficulty eating and drinking or abdominal pain underwent thoracic or peritoneal

5 drainage for symptomatic relief. Hepatorenal syndrome was diagnosed according to the criteria of the

7 International Ascites Club [5] as follows: 1) low glomerular filtration rate, indicated by

8 serum creatinine $>1.5 \mathrm{mg} / \mathrm{dl}$ or 24 -h creatinine clearance $<40 \mathrm{ml} / \mathrm{min} ; 2$ ) absence of

9 shock, ongoing bacterial infections, and recent or current treatment with nephrotoxic

10 drugs; 3) no sustained improvement of renal function by diuretic withdrawal and

11 intravenous administration of fluids; and 4) absence of significant proteinuria $(<500$

$12 \mathrm{mg} /$ day) and ultrasonographic abnormalities in the kidneys.

13 Infections and bacteremia were defined using the criteria proposed by the

14 Centers for Disease Control and Prevention and based on our previous report regarding 15 LT patients [2]. Diagnosis of infection in ascitic fluid or pleural effusion, including 16 spontaneous bacterial peritonitis (SBP) and spontaneous bacterial empyema (SBEM), 17 was based on the level of polymorphonuclear white cells $\left(>250 / \mathrm{mm}^{3}\right.$ with positive 18 culture or $>500 / \mathrm{mm}^{3}$, if culture was negative) $[6,7]$. 
Thoracic and intraperitoneal drainage were not indicated as the treatment of

$2 \quad$ SBEM and SBP in general, instead antimicrobial therapy was started immediately. In

3 cases where SBEM and SBP had been diagnosed after the initiation of drainage,

4 drainage was maintained in combination with antimicrobial therapy.

5

The study protocol was approved by the Medical Ethics Committee of Kyoto

6 University, and the study was performed in accordance with the ethical standards

7 established in the 1975 Declaration of Helsinki.

8

\section{$9 \quad$ Drainage protocol}

10 We used fine catheters (Argyle ${ }^{\mathrm{TM}}$ aspiration Seldinger kit, 5Fr; COVIDIEN Japan,

11 Shizuoka, Japan) for thoracic and intraperitoneal drainage to avoid injuring collateral

12 vessels. Before performing drainage, Doppler ultrasonography and CT were used to

13 establish the location of any abnormal intercostal or abdominal wall collateral vessels so

14 as to avoid hemorrhage. To prevent hypotension, depletion of protein and electrolytes,

15 and re-expansion pulmonary edema rapid drainage of ascites and pleural effusion was

16 avoided. Initially, $1000 \mathrm{ml}$ was drained, then the drainage volume was gradually

17 increased from the second day. At the same time, the intravenous fluid and albumin

18 replacement was undertaken during the drainage. 


\section{$1 \quad$ Statistical analysis}

2 Values are presented as means and standard deviations (SD) unless otherwise indicated.

3 Continuous data were analyzed by Student's $t$-test or the Mann-Whitney test, while

4 categorical data were analyzed with the chi-square test. For survival analyses,

5 Kaplan-Meier survival curves were constructed and analyzed by the log rank test, and

6 multivariate analyses of survival were performed by proportional regression hazard

7 analyses. Variables identified as significant $(P<0.05)$ in univariate analyses were

8 considered to be candidates for the multivariate analyses. Values of $P<0.05$ were

9 considered significant. Statistical analyses were performed using Prism version 5

10 (GraphPad Software Inc., San Diego, USA) for the univariate analyses and JMP version

119 (SAS institute Inc., Cary, NC, USA) for the multivariate analyses. 


\section{RESULTS}

2

3 Details of Preoperative Drainage Management

4 Preoperative thoracic or intraperitoneal drainage was performed in 36 cases (15.2\%), all

5 later underwent scheduled living-donor LT. In 16 cases thoracic drainage was required

6 (including five cases with SBEM), intraperitoneal drainage was performed in 15 cases

7 (including eight cases of SBP); and both thoracic and intraperitoneal drainage was

8 needed in five cases (including two cases of SBP). The median drainage period was 13

9 days (range: 1-33 days) for thoracic drainage and 9 days (1-44 days) for intraperitoneal 10 drainage.

11 Infectious complications related to the placement of an intraperitoneal 12 drainage tube occurred in three patients (8.3\%), while there were no complications 13 related to the placement of a thoracic drainage tube. Infection was diagnosed 2, 6, and 7 14 days after placement of the drainage catheter; patients were treated with antibiotics and 15 a new catheter was resited. There were no other complications, such as hemothorax or 16 pneumothorax.

\section{$18 \quad$ Patient characteristics}

19 Table 1 shows the characteristics of the groups: there were no significant differences in 20 the sex, recipient age, blood type compatibility, graft-recipient weight ratio (GRWR), 21 operation time, cold and warm ischemic times, model for end-stage liver disease 22 (MELD) score, evidence of preoperative hepatic encephalopathy, and preoperative 23 serum creatinine of the patients in each group. Group HA was characterized by higher 24 intraoperative blood loss $(P=0.02)$, higher Child-Pugh score $(P=0.001)$, lower 
1 preoperative serum albumin concentration $(P=0.01)$, and higher serum blood urea

2 nitrogen concentration $(P=0.003)$ compared with group C.

3

\section{$4 \quad$ Preoperative $\mathrm{HRS}$ and perioperative infections}

5 Group HA had a significantly higher incidence of HRS than group C (nine out of 36

6 cases [25\%] versus 20 out of 201 cases [9.9\%], $P=0.017$ ), and a significantly higher

7 incidence of preoperative infection (19 out of 36 cases [52.8\%] versus 35 out of 201

8 cases [17.4\%], $P=0.0001)$. The incidence of bacteremia within 90 days of LT was

9 significantly higher in group HA than group C (20 out of 36 cases [55.6\%] versus 94 10 out of 201 cases [46.7\%], $P=0.008)$.

\section{Postoperative mortality}

13 Figure 1 shows the Kaplan-Meier survival curves of each group. The cumulative 14 survival rates at 1 and 3 years after LT were both significantly lower in group HA than 15 group C (1 year survival: 58.9\% versus $82.9 \%$; 3 year survival: $58.9 \%$ versus $77.7 \%$, 16 respectively; $P=0.003)$. Survival was worse in the HA group whether the patient had 17 undergone intrathoracic or intraperitoneal drainage (Fig. 2a,b). Even when cases of 18 infectious H\&MA were excluded, those with sterile preoperative H\&MA ( $n=23)$ had a 19 significantly worse prognosis than those in group C (1 year survival: 64.6\% versus $20 \quad 88.1 \%$; 3 year survival: 64.1\% versus 82.6\%, respectively; $P=0.015$; Fig. 3).

21 When we subdivided the patients in group HA into two groups depending on 22 the diagnosis of postoperative bacteremia, we found that 1- and 3-year survival rates 23 were significantly reduced in those with bacteremia compared with those who were not 24 (1-year survival: 41.2\% versus 86.2\%; 3-year survival: $41.2 \%$ versus $86.2 \%$, 
1 respectively; $P=0.008)$.

2

3 Prognostic indicators after $\mathbf{L T}$

4 Blood loss, Child-Pugh score, preoperative albumin and blood urea nitrogen 5 concentrations, HRS, preoperative infection, and GRWR, were included in the 6 multivariate analysis along with preoperative uncontrollable H\&MA. We found that 7 preoperative uncontrollable H\&MA (hazard ratio: 2.304; $P=0.034$ ) and Child-Pugh 8 score (hazard ratio: $1.258 ; P=0.003$ ) were independent risk factors for mortality after $9 \quad$ LT (Table 2).

10 


\section{DISCUSSION}

2

3 We analyzed the incidence and characteristics of patients with uncontrollable H\&MA

4 before LT and evaluated its effect on the postoperative course after LT.

5

6 diaphragmatic defect. Therefore, we included patients with hepatic hydrothorax and

7 those with massive ascites in the same group. When we subdivided these patients into

8 two groups based on the type of drainage, the survival rates were almost the same.

9 Ascites is attributed to impaired albumin production in the liver, portal hypertension,

10 and salt retention owing to renal dysfunction. These symptoms are usually treated with

11 a high-protein diet and diuretics, but in some cases ascites proves to be refractory to manipulating the dose and type of diuretics and dietary intake. A vicious cycle may

13 develop in which increasing abdominal distension further impairs hepatic function.

14 Ascites and hydrothorax can cause SBP and SBEM, respectively, and can also cause a

15 decrease in the circulating blood volume, which can lead to HRS. A transhepatic

16 intra-jugular porto-systemic shunt (TIPS) is one of the options for treating refractory

17 hepatic hydrothorax and massive ascites, and there are reports that TIPS is superior to 18 large-volume paracentesis in the control of ascites or hydrothorax [8,9]; however, it 19 only provides supportive care and cannot prolong survival.

It is well recognized that the health of an LT recipient pre-transplant is closely

21 associated with postoperative mortality. Our study showed that patients with preoperative uncontrollable H\&MA had a higher mortality rate after LT. The causes of death were mainly related to postoperative infections, including bacteremia. Notably,

24 when cases of infectious H\&MA were excluded, the remaining recipients with 
1 uncontrollable H\&MA still had a poorer survival than those in group C. This finding

2 suggests that LT recipients with uncontrollable H\&MA are at risk of post-transplant

3 mortality regardless of the presence of preoperative infection. It is likely that the

4 recipients in group HA were more severely compromised by more severe end-stage

5 liver disease. The higher rate of postoperative infections in group HA might also be a

6 consequence of poorer general condition and comorbidities of the patients with high

7 Child-Pugh scores. The substantially reduced survival rates in patients in group HA

8 diagnosed with postoperative infections suggests that effective postoperative infection

9 control could be a crucial means of improving outcome after LT.

10 MELD scores were not substantially different between the groups in our study.

11 MELD score is a useful means of prioritizing the waiting list, but it is controversial as

12 to whether it can effectively predict survival after LT [10-12]. The severity of

13 preoperative ascites and hepatic hydrothorax, which are not sufficiently reflected in the

14 MELD score, might have a greater impact on survival. Clinicians should carefully

15 consider the timing of LT, undertaking transplantation - where possible - before

16 H\&MA becomes uncontrollable.

17 The multivariate proportional hazard analyses revealed that uncontrollable

18 H\&MA was an independent risk factor for postoperative mortality in our study.

19 Somsouk et al. [13] reported that patients with moderate ascites and a MELD score <21

20 were at higher risk of death while on the waiting list for LT. It is possible that the

21 presence of preoperative uncontrollable H\&MA may be a more important prognostic

22 indicator than the MELD scores.

Xiol et al. [14] and Serste et al. [15] have reported that the presence of

24 preoperative hepatic hydrothorax had no significant negative influence on postoperative 
1 outcome after deceased donor LT. Xiol et al. [14] reported that the survival rate of

2 patients with hydrothorax was $70 \%$ at 8 years. However, in their hydrothorax group,

3 they included not only patients with refractory hydrothorax but also those with previous

4 episodes of spontaneous bacterial empyema and those with uncomplicated hydrothorax

5 with impaired hepatic function. In addition, the Child-Pugh score in their hydrothorax

6 group was 9.9 \pm 1.4 , which was lower than that in our study (mean: 11.5). Serste et al.

7 [15] established two control groups: a group with ascites but not hydrothorax; and a

8 group with no ascites or hydrothorax, and compared survival between the three groups.

9 They found no significant difference in the overall risk of death, but the 1-year survival

10 rate in the hydrothorax group was $64 \pm 15 \%$, which is higher than expected. The apparent

11 discrepancy in findings of the impact of hydrothorax may also be a consequence of the type of LT. Most of the cases in Xiol and Serste’s studies [14,15] were deceased donor

13 LT cases, while all our cases received grafts from living donors. As the graft volume is

14 limited in living-donor LT, H\&MA may have persisted due to higher portal venous 15 pressures and hypoalbuminemia due to inadequate postoperative hepatic synthetic 16 function.

17 It has still not been established whether thoracic and/or intraperitoneal drainage is 18 the best means of managing uncontrollable H\&MA for liver cirrhosis (LC) before 19 scheduled LT. However, complications related to paracentesis have been reported in 20 only about $1 \%$ of patients with coagulopathy [16], therefore intraperitoneal drainage 21 appears to be a safe approach. According to treatment guidelines for LC $[17,18]$, 22 intraperitoneal drainage is an effective first line treatment for uncontrollable tense and 23 refractory ascites. Total paracentesis reduces intra-abdominal, intrathoracic, right 24 arterial, and pulmonary pressures, improving cardiac output by increasing stroke 
1 volume without changing heart rate [19]. Moreover it results in a rapid fall in portal

2 pressure, by decreasing the wedged hepatic venous pressure and hence the hepatic

3 venous pressure gradient [20]. Although intraperitoneal drainage is an established

4 treatment for uncontrollable massive ascites, there are no data on its role in the

$5 \quad$ management of SBP [18].

6 Regarding the management of refractory hydrothorax, thoracic drainage using a

7 chest tube should be avoided due to the risk of complications [21]. It has been reported

8 that chest tube insertion for hepatic hydrothorax carries significant morbidity and

9 mortality, with questionable benefit [22,23]. In our institution, however, the morbidity

10 was $8.3 \%$, all related to intraperitoneal drainage, and we experienced no serious or fatal

11 complications such as hemothorax or pneumothorax.

12 Drainage of H\&MA might adversely influence a patient's preoperative condition.

13 Drainage of fluid could cause electrolyte and hemodynamic disturbance, and impair

14 renal function. This can be prevented by adequate volume replacement with an

15 appropriate combination of intravenous fluids. Nevertheless, antibodies and immune

16 competent cells in the hydrothorax and ascitic fluid cannot be replaced, which might

17 result in a state of relative immunodeficiency and increase the rate of postoperative 18 infections.

19 Thoracic and intraperitoneal drainages alone without LT will not improve the 20 prognosis of patients with end-stage liver diseases obviously, but we should aim to 21 improve the recipient's condition as much as possible before LT, especially if in living 22 donor procedures. Recently, we have ensured that drainage of ascites or hydrothorax is 23 not undertaken in the 3 days before LT in an effort to avoid intraperitoneal infections. 
1 study, and the numbers of patients are small. We believe that a larger series and a

2 multicenter study design would address these issues.

3 In conclusion, uncontrollable H\&MA was found to be an independent risk

4 factor for poor post-transplant outcome in our study. In particular, for patients with

5 uncontrollable H\&MA, effective postoperative treatment of infection is key to

6 improving outcome after LT. In addition, the timing of transplant is crucial; efforts

7 should be made to undertake surgery before H\&MA becomes uncontrollable.

8

9 


\section{Conflict of interest:}

2 None of the authors has a conflict of interest to declare.

3

4 


\section{References}

2 1. Taketomi A, Shirabe K, Toshima T, Hashimoto N, Kayashima H, Ikegami T, et al.

3 The long-term outcomes of patients with hepatocellular carcinoma after living donor

4 liver transplantation: a comparison of right and left lobe grafts. Surg Today.

$5 \quad 2012 ; 42: 559-64$

6 2. Iida T, Kaido T, Yagi S, Yoshizawa A, Hata K, Mizumoto M, et al. Post-transplant

7 bacteremia in adult living donor liver transplant recipients. Liver Transpl.

$8 \quad 2010 ; 16: 1379-85$.

9 3. Arroyo V, Gines P, Gerbes AL, Dudley FJ, Gentilini P, Laffi G, et al. Definition and 10 diagnostic criteria of refractory ascites and hepatorenal syndrome in cirrhosis. 11 International Ascites Club. Hepatology. 1996;23:164-76.

12 4. Morrow CS, Kantor M, Armen RN. Hepatic hydrothorax. Ann Intern Med. 13 1958;49:193-203.

14 5. Gines P, Arroyo V. Hepatorenal syndrome. J Am Soc Nephrol. 1999;10:1833-9.

15 6. Rimola A, Garcia-Tsao G, Navasa M, Piddock LJ, Planas R, Bernard B, et al. 16 Diagnosis, treatment and prophylaxis of spontaneous bacterial peritonitis: a consensus 17 document. International Ascites Club. J Hepatol. 2000;32:142-53.

18 7. Xiol X, Castellvi JM, Guardiola J, Sese E, Castellote J, Perello A, et al. Spontaneous 19 bacterial empyema in cirrhotic patients: a prospective study. Hepatology. 20 1996;23:719-23.

21 8. Salerno F, Merli M, Riggio O, Cazzaniga M, Valeriano V, Pozzi M, et al. Randomized 22 controlled study of TIPS versus paracentesis plus albumin in cirrhosis with severe 23 ascites. Hepatology. 2004;40:629-35.

24 9. Sanyal AJ, Genning C, Reddy KR, Wong F, Kowdley KV, Benner K, et al. The North 
1 American Study for the Treatment of Refractory Ascites. Gastroenterology.

$2 \quad 2003 ; 124: 634-41$.

3 10. Hayashi PH, Forman L, Steinberg T, Bak T, Wachs M, Kugelmas M, et al. Model for

4 End-Stage Liver Disease score does not predict patient or graft survival in living donor

$5 \quad$ liver transplant recipients. Liver Transpl. 2003;9:737-40.

6 11. Ishigami M, Honda T, Okumura A, Ishikawa T, Kobayashi M, Katano Y, et al. Use

7 of the Model for End-Stage Liver Disease (MELD) score to predict 1-year survival of

8 Japanese patients with cirrhosis and to determine who will benefit from living donor

9 liver transplantation. J Gastroenterl. 2008;43:363-8.

10 12. Selzner M, Kashfi A, Cattral MS, Selzner N, McGilvray ID, Greig PD, et al. Ann 11 Surg. 2010;251:153-7.

12 13. Somsouk M, Kornfield R, Vittinghoff E, Inadomi JM, Biggins SW. Moderate ascites 13 identifies patients with low model for end-stage liver disease scores awaiting liver 14 transplantation who have a high mortality risk. Liver Transpl. 2011;17:129-36.

15 14. Xiol X, Tremosa G, Castellote J, Gornals J, Lama C, Lopez C, et al. Liver 16 transplantation in patients with hepatic hydrothorax. Transpl Int. 2005;18:672-5.

17 15. Serste T, Moreno C, Francoz C, Razek WA, Paugham C, Belghitti J, et al. The 18 impact of preoperative hepatic hydrothorax on the outcome of adult liver transplantation. 19 Eur J Gastroenterol Hepatol. 2010;22:207-12.

20 16. Runyon BA. Paracentesis of ascitic fluid. A safe procedure. Arch Intern Med. 21 1986;146:2259-61.

22 17. Runyon BA, Committee APG. Management of adult patients with ascites due to 23 cirrhosis: an update. Hepatology. 2009;49:2087-107.

24 18. Moore KP, Aithal GP. Guidelines on the management of ascites in cirrhosis. Gut. 
$1 \quad$ 2006;55 Suppl 6:vi1-12.

2 19. Pozzi M, Osculati G, Boari G, Serboli P, Colombo P, Lambrughi C, et al. Time

3 course of circulatory and humoral effects of rapid total paracentesis in cirrhotic patients

4 with tense, refractory ascites. Gastroenterology. 1994;106:709-19.

5 20. Luca A, Feu F, Garcia-Pagan JC, Jimenez W, Arroyo V, Bosch J, et al. Favorable

6 effects of total paracentesis on splanchnic hemodynamics in cirrhotic patients with tense

7 ascites. Hepatology. 1994;20:30-3.

8 21. Cardenas A, Arroyo V. Management of ascites and hepatic hydrothorax. Best Pract

$9 \quad$ Res Clin Gastroenterol. 2007;21:55-75.

10 22. Orman ES, Lok AS. Outcomes of patients with chest tube insertion for hepatic

11 hydrothorax. Hepatol Int. 2009;3:582-6.

12 23. Liu LU, Haddadin HA, Bodian CA, Sigal SH, Korman JD, Bodenheimer HC, Jr., et

13 al. Outcome analysis of cirrhotic patients undergoing chest tube placement. Chest. $14 \quad 2004 ; 126: 142-8$. 


\section{Tables:}

2

3

4

TABLE 1. Background and Characteristics of the Two Groups

\begin{tabular}{lccc}
\hline & Group HA $(n=36)$ & Group C $(n=201)$ & $P$ \\
\hline Sex (male/female) & $18 / 18$ & $99 / 102$ & 0.54 \\
Age & $55.3 \pm 7.89$ & $51.1 \pm 12.81$ & 0.05 \\
ABO compatibility & identical/compatible 28 & identical/compatible 153 & 0.51 \\
& incompatible 8 & incompatible 48 & \\
Graft type & right 15, left 19 & right 114 , left 71 & 0.08 \\
& posterior 2 & posterior 6 , whole 10 & \\
GRWR & $0.90 \pm 0.17$ & $0.98 \pm 0.31$ & 0.14 \\
Operation time (min) & $814.8 \pm 120.5$ & $793.6 \pm 149.4$ & 0.45 \\
Blood loss (ml) & $12244.2 \pm 9505.48$ & $8814.4 \pm 7251.1$ & 0.02 \\
CIT (min) & $98.1 \pm 53.3$ & $119.1 \pm 113.0$ & 0.31 \\
WIT (min) & $43.6 \pm 13.2$ & $48.6 \pm 53.4$ & 0.60 \\
MELD score & $20.9 \pm 8.84$ & $20.5 \pm 9.47$ & 0.79 \\
Child-Pugh score & $11.4 \pm 2.20$ & $10.0 \pm 2.22$ & 0.001 \\
& $1.44 \pm 0.65$ & $1.32 \pm 0.65$ & 0.1517 \\
Preop hepatic encephalopathy & $2.65 \pm 0.42$ & $2.94 \pm 0.52$ & 0.001 \\
Preop serum Alb (g/dl) & $28.9 \pm 19.4$ & $18.9 \pm 14.3$ & 0.0003 \\
Preop serum BUN (mg/dl) & $1.17 \pm 0.68$ & $1.19 \pm 3.58$ & 098 \\
Preop serum Cr (mg/dl) & & & \\
\hline
\end{tabular}

GRWR. graft-recipient weight rate; CIT, cold ischemic time; WIT, warm ischemic time; MELD, model for endstage liver disease; Preop, preoperative; Alb, albumin; $\mathrm{BUN}$, blood urea nitrogen; $\mathrm{Cr}$, creatinine. 
1

TABLE 2. Independent Prognostic Risk Factors for mortality after liver transplantation

\begin{tabular}{lccc}
\hline Variable & Hazard ratio & $\begin{array}{c}95 \% \text { Confidence } \\
\text { interval }\end{array}$ & $P$ \\
\hline $\begin{array}{l}\text { Preop uncontrollable } \\
\text { hydrothorax and massive ascites }\end{array}$ & 2.304 & $1.069-4.691$ & 0.0339 \\
Blood loss & 1.000 & $0.999-1.000$ & 0.1123 \\
Child-Pugh score & 1.258 & $1.085-1.422$ & 0.0033 \\
Preop serum Alb & 1.003 & $0.460-2.144$ & 0.9938 \\
Preop serum BUN & 1.004 & $0.983-1.024$ & 0.7006 \\
HRS & 1.345 & $0.434-3.633$ & 0.5884 \\
Preop infection & 1.088 & $0.493-2.270$ & 0.8281 \\
GRWR & 0.971 & $0.269-3.036$ & 0.9612 \\
\hline
\end{tabular}

Preop, preoperative; H\&MA, hydrothorax and massive ascites; Alb, albumin; BUN, blood urea nitrogen; GRWR, graft-recipient weight ratio. 


\section{$1 \quad$ Figure Legends}

2 Figure 1. Survival rates of patients with (group HA) and without (group C)

3 uncontrollable H\&MA. The cumulative survival rates after LT were significantly lower

4 in group HA than group $\mathrm{C}(P=0.0007)$.

5

6 Figure 2. Survival rates of patients depending on the site of drainage. Patients who

7 underwent intraperitoneal drainage (a) and those who underwent thoracic drainage (b)

8 had higher mortality rates than those in group C.

9

10 Figure 3. Kaplan-Meier curves comparing group HA and group C having excluded

11 infectious H\&MA cases from group HA. Mortality rates are still significantly higher in 12 group HA than group C. 


\section{Figures:}

\section{Fig.1:}

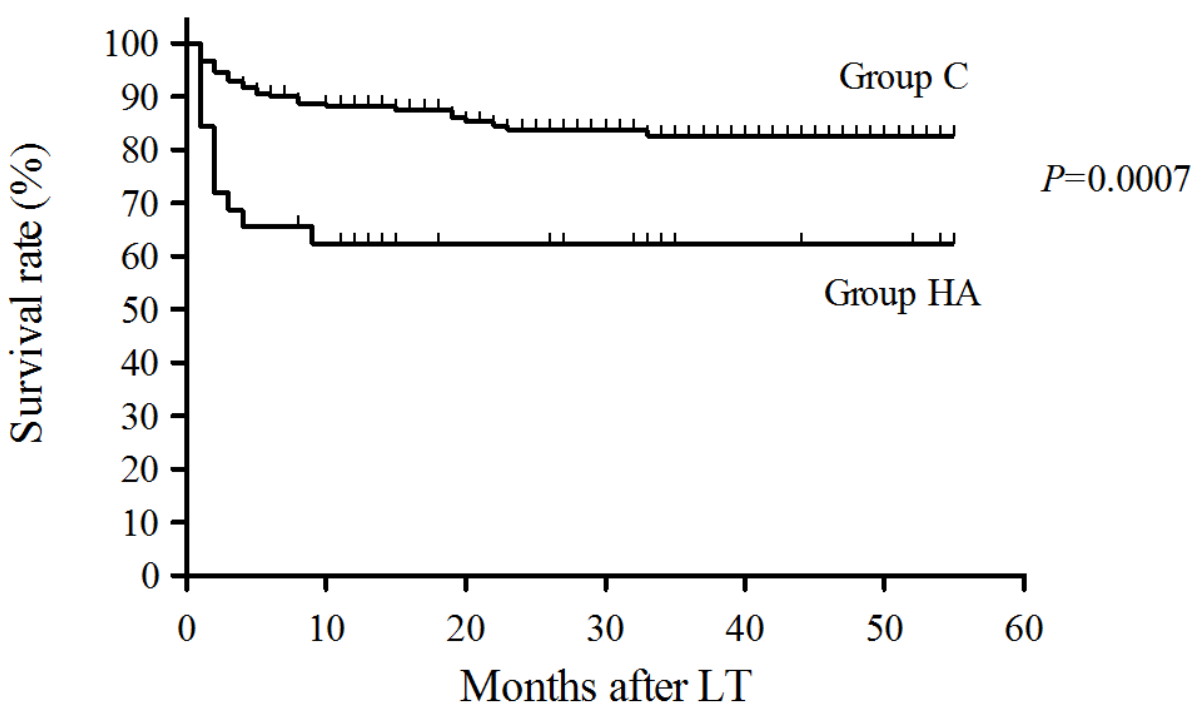

3

4 Fig.2:

a

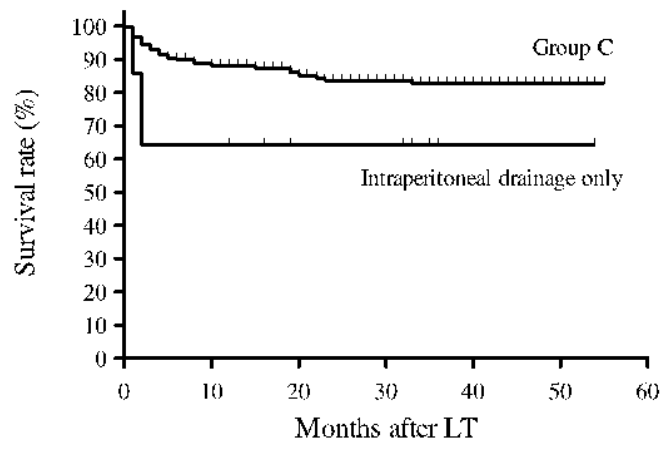

$P=0.0259$ b

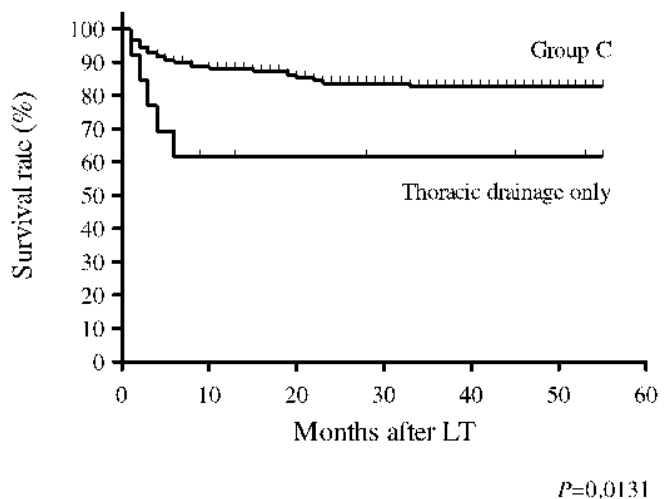




\section{$1 \quad$ Fig.3:}

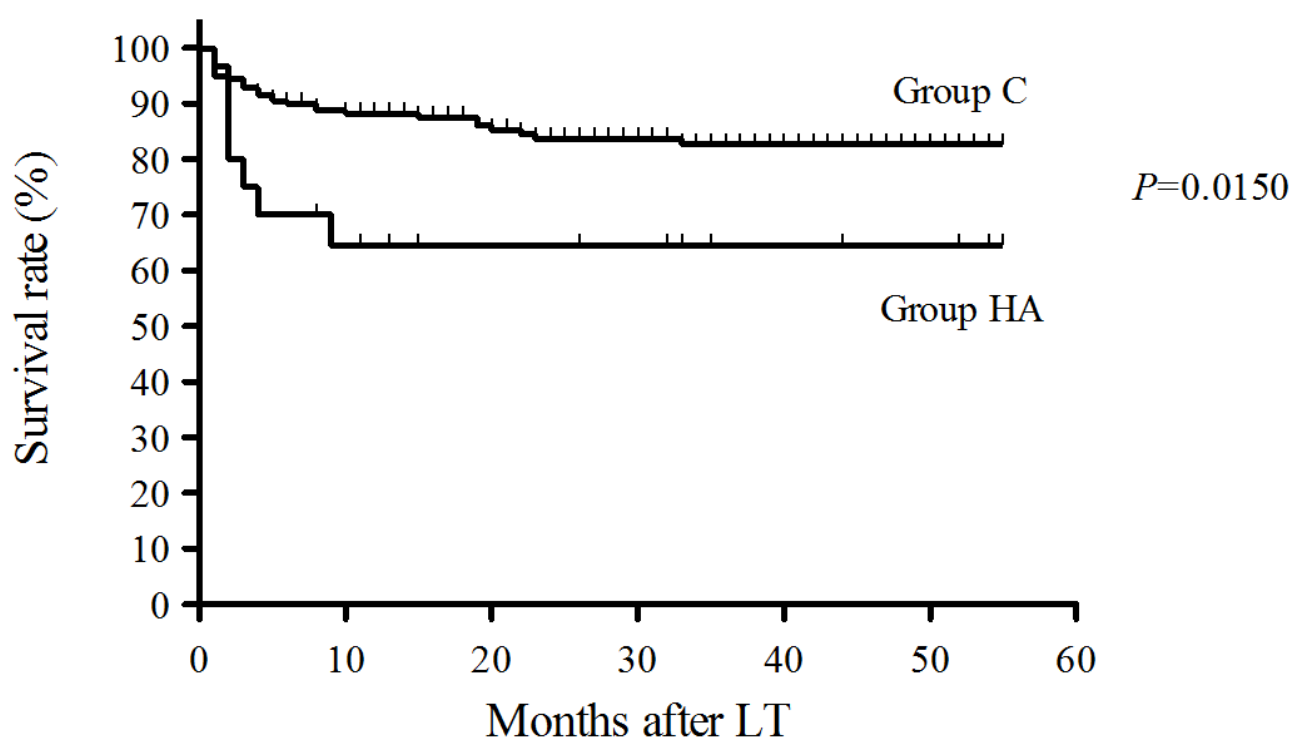

